\title{
Research on the Hierarchical Teaching Method of University English Based on Artificial Intelligence Wireless Network
}

\author{
Qian Zhang ${ }^{1}$ and Zeyu Ji $\mathbb{C}^{2}$ \\ ${ }^{1}$ Prospect College, Jinzhong College of Information, Jinzhong 030800, China \\ ${ }^{2}$ Department of Computer Science, Xi'an Jiaotong University, Xi'an 710049, China
}

Correspondence should be addressed to Zeyu Ji; zeyu.ji@stu.xjtu.edu.cn

Received 28 October 2021; Accepted 15 December 2021; Published 25 February 2022

Academic Editor: Narasimhan Venkateswaran

Copyright (C) 2022 Qian Zhang and Zeyu Ji. This is an open access article distributed under the Creative Commons Attribution License, which permits unrestricted use, distribution, and reproduction in any medium, provided the original work is properly cited.

\begin{abstract}
University English is one of the basic core courses for all university students. The course involves many difficult concepts and grammatical structures, so it is difficult to most students. The starting point in any teaching program is to determine whether teaching is needed to specify what that teaching should accomplish. So the students' need is of vital importance to the success of university English teaching. Traditional university English teaching does not take into account students' individual learning abilities and feedback, which would cause students to not grasp enough the key knowledge, and then make students lose their interest in English. The development of artificial intelligence technology is becoming more and more mature, especially in the application of English teaching, which promotes the reform, development, and modernization of English teaching. Hence, in this paper, we propose a hierarchical teaching method for the university English teaching platform and employ artificial intelligence to find the needs of university students and know about the mastering knowledge of students. Initially, the dataset is preprocessed using normalization, and then, the feature extraction is performed using principal component analysis (PCA). For classification of the data, we employ the $K$-means clustering algorithm. To enhance the evaluation system, the whale optimization algorithm (WOA) is used. The performance of the proposed system is analyzed, and it is shown that the average score of students who used the proposed platform to learn university English is far higher than those students who did not use the platform. Hence, the platform can improve students' autonomous learning and English abilities.
\end{abstract}

\section{Introduction}

The traditional method of teaching English needs to be improved. The students are the major concern for the change in the standard of teaching methods, and the teachers became leaders to guides. The emergence of the Internet and multimedia classroom in colleges and universities has changed the way of teaching from a closed environment to an open environment with intelligence, network, and digitalization. Thus, the traditional English teaching methods needs to be changed by the English teachers by using modern information technologies and developing new reforms in the direction of English teaching. Traditional methods of teaching English cannot keep up with the rapid technological changes and society. It is time to create a new teaching standard. In recent times, a number of enhanced techniques for English teaching have been suggested by various academics in an attempt to increase the quality and level of English instruction [1].

University English is not only a language course that gives basic knowledge about English but also a capacity development course that supports students to broaden their horizons and learn about different cultures in the world. The University English course should be designed to provide students with a solid foundation in the English language and the ability to use and communicate English effectively. It should encourage students' individual learning progress and provide a gradual growth in their English proficiency so as to meet the needs of their development in different specialties. The development of the curriculum should be based on the analysis of student necessities and their lacking skills. The designed curriculum should also 
improve the student to be proficient in the English language [2].

The students are the major focus of education; the new learning methods and the constructive teaching theory are becoming increasingly popular at present times. The distributed characteristics and strong interaction of the computer network platform and the development of computer technology provide technical feasibility for personalized learning. The personalized learning phase provides all kinds of resources which are suitable for every individual irrespective of their time and place, learning abilities, different cultural backgrounds, and their learning styles, skills, and speed of learning. The personalized individual services are an essential service provided for every student to identify their strength and weakness and to enhance their full development process. It is also the only way to realize the education information under the condition of modern information technology [3].

An artificial intelligence platform that may be used for both teaching and assessing student performance. It may be tailored to meet the needs of students at every level of study. The AI platform is built using key AI schemes such as identification and pattern recognition, decision-making process and choosing, theoretical connection and reasoning, and implementation and sequential management, as well as scheduling and solving problems. Knowledge-based systems and supervised machine learning methods are utilised to assess student achievement [4].

In order to attract student's attention in the educational sector, new methodologies have been implemented. The best courses on each subject are also provided by web-based engines. In English education, artificial intelligence (AI) is a difficult and innovative subject. It teaches and promotes a judgment-free global educational atmosphere. The aim is to study outside of the classroom to enhance technology throughout one's life. Classrooms are becoming more globally interconnected and accessible as a result of their worldwide introduction. In order to develop intelligent teaching programs, AI is used to monitor learner's mental activities such as self-regulation, management, and descriptions. This selects the most appropriate information for the learner. AI assists in the transformation of knowledge and training into deep thinking and learning techniques. Artificial educational intelligence is helping to foster technical innovation and social awareness [5].

The further part of this article is structured as shown. Section 2 offers the literary works associated with this paper. Section 3 explains the proposed model. Section 4 provides the performance analysis of the suggested method. And finally, Section 5 concludes the overall idea of the paper.

\section{Related Works}

(i) Zhao and $Y u$ [6]: a thorough study of the wireless network's design and modelling is carried out, and it serves as the basis for future theoretical innovation. In wireless network remote monitoring technology, the traditional Gauss mixture model method is utilised for monitoring anoma- lous targets and object movements to address the problems of target loss and confusion, and it is tracked successfully. The suggested algorithm flow is validated via simulation and experimental examination of the method using the AVR software system in the experimental section.

(ii) $\mathrm{Li}$ et al. [7]: a model of a collegiate English mobile learning system based on a CAD model is presented, and the structure of the model is described, as well as different functional modules. It creates a series of dependable IoT node information collecting systems, employs data collection techniques and node information reliability assessment methods, and determines the node information reliability based on system data. The development of a reliable environmental data collection system and a technique for assessing the trustworthiness of node information is completed. This enhances the Internet of Things security monitoring system as well as the efficiency with which English may be learned.

(iii) Juan and Hong Wei [8]: it is easy to fall victim to the issue of local extreme value and sluggish convergence speed while training state neural networks. The particle swarm optimization technique is used to build the new teaching approach for English schools, and it is integrated into the neural network algorithm. The RNN method may be used to search for both continuous and target functions worldwide. It employs effective global search features. The back propagation method was used to address the limitations of the BP algorithm. It takes a long training time of the neural network and slow convergence because of a lot of gradient calculations. The CNN algorithm and the standard $\mathrm{BP}$ algorithm have a comparative experimental analysis. However, the efficiency of neural network training can be improved by using the $\mathrm{CNN}$ algorithm. The student's English learning ability can be estimated by the $\mathrm{CNN}$, and the neural network training is provided. The training and test analysis results have been implemented for a complex classification problem, and it shows the efficiency of the model. This method may satisfy the requirements of instructors and students for multidirectional interaction and consultation while also achieving scientific and efficient educational objectives. Each connection promotes deep learning by maximizing the development of a student's autonomous learning capacity and marching toward higher-order creative abilities.

(iv) Chen [9]: the technique of calculating an RBF regularization network was used to provide the knowledge fusion and optimization of the RBF neural net selection method for the performance assessment system of college English teaching. It employs an experimental research technique and 
an RBF neural network decision algorithm. The evaluation method may be used to quantify the current state of English instruction. Teachers may identify the issues in teaching and enhance the teaching quality by measuring the teaching outcomes and evaluating the causes.

(v) Lou [10]: this work attempts to allocate and manage the resource efficiently for English teaching. Initially, English teaching resources are verified, and the frequency-inverse document frequency (TF-IDF) weight method and $k$-nearest neighbor $(\mathrm{KNN})$ algorithm were used to improve the efficiency and allocate the resources. Through case analysis, the improved methods were verified. The results show that to allocate English teaching resources, KNN provides a feasible way. The research findings provide reference to the storage and allocation of teaching resources.

(vi) Zhang [11]: this work incorporated multimedia teaching in the English classroom teaching, and it helps to stimulate student interest in learning and improve their learning efficiency. Through the way of human-computer interaction, people's body movements and gesture information to communicate the traditional teaching mode have been changed. To extract the feature value of the vector angle through the three-dimensional characteristics of people's bones, the AI technology is used and the KNN rapid recognition method is proposed. The SSH framework and the C/S structure is used for the English teaching system, and the patterns are designed for the software reusability. Finally, functional test and performance tests were performed on the system. The results show that this system can meet the needs of teaching and assist teaching in English

(vii) Guo [12]: this work investigated behavior analysis as a breakthrough point for teaching English. To compare the $K$-nearest neighbor algorithm and the support vector machine algorithm, it utilises the determining methods on the basis of inductive analysis and optimizes the plan analysis methods for teaching English by combining big data mining technology. A classification prediction model is built. The prediction of English teaching integration is optimized through the built prediction model. The classifier predicts the results for accuracy and precision. This can provide a basis for educators to optimize decision-making and teaching methods.

(viii) Li [13]: intelligent English education has emerged as an unavoidable tendency in the evolution of future English teaching models, necessitating collaboration with intelligent text recognition technologies. The convolutional neural network method based on SVM is used to recognise text in English instructional material. After feature extraction, the original text content was transformed into data, which was then utilised to identify and analyze the machine. The performance of the method is evaluated using example verification. It demonstrates that the suggested method's result has a high accuracy rate and can be used to classify text in English teaching material and offer a reference point for relevant research.

(ix) Jiang [14]: this work investigated student behavior and academic data for interpreting the data inherent patterns and correlations. The patterns are logically utilised to offer direction for teaching activities and management and to enhance the quality of teaching monitoring. Technologies are utilised to improve the monitoring of the department of the company and to provide a timely alert system for teacher training college management. As a result, counsellors can make informed decisions and offer early assistance to kids with repeated warnings. The genetic algorithm is used to create a GABP hybrid prediction model to address the local minimum issue of the $\mathrm{BP}$ neural network method. The data validation score is 95 percent, while the F1 score is about 86 percent, indicating that the algorithm prediction results are much more accurate. It provides college administrators with reliable data to forecast retention. Finally, appropriate recommendations are made, and issues with the retention prediction model's implementation are examined.

(x) Shuoyan [15]: this work presents an English language education teaching model for college students. The network technology suggested is dependent on the proposal of the education platform. The requirements of current social development and college student's comprehensive English ability for English teaching are promoted.

(xi) Quan [16]: presented for training, we developed a machine learning prediction and artificially intelligent KNN method. Individual characteristics from group feature recognition, as well as student emotion recognition, were identified using instructional pictures. The experimental findings show that the suggested technique has certain impacts and may be utilised as an approach to aid in the teaching and learning process as well as a theoretical reference for future study.

(xii) Dan [17]: this work provided a research of the symmetric SDAE collaborative filtering algorithm which was conducted in order to build an intelligent English resource recommendation system. The symmetric SDAE model is made up of two stacked denoising automated codes, one for training item information and the other for training user information and obtaining the user feature 
matrix. Simultaneously, in order to investigate the model's performance, this article analyzes expert and student user evaluations, applies mathematical statistics and machine learning to data processing, and presents the findings in graphs. The study findings indicate that the model developed in this article produces excellent outcomes, and a new research path is suggested.

(xiii) Wanni [18]: a sophisticated basic grammar detection system was built using a mix of evolutionary and KNN algorithms. This article proposes a grammatical mistake correction model based on rules and statistics, based on advanced theoretical and technological expertise. In addition, this research offers an error correction model of linked word grammar for the relevant related word mistakes. Furthermore, the characteristics of artificially generated English-related words are chosen for error correction, and the best feature subset is chosen to enhance model error correction accuracy.

(xiv) Quan [19]: this work proposed a machine learning prediction and artificially intelligent $\mathrm{KNN}$ algorithm. Furthermore, this research gathers videos and instruction pictures for student feature detection and recognition, differentiates specific elements from accurate depiction, and identifies student school of communication in great detail. This research also created a case study to assess the algorithm's effectiveness. The experimental findings show that the suggested algorithm has specific impacts and may be utilised as a teaching aid as well as a theoretical foundation for future study.

(xv) Ran et al. [20]: the issue of phonetic mistake correction at the phoneme level was addressed. The fundamental method of voice cutting is primarily described, covering the front-end analysis of the voice and the extraction of feature variables, after the presentation of basic understanding, building, and learning of an ANN model. Following the appropriate processing of the speech signal, voice cutting according the acoustic model and the built network structure is required, as well as error variance judgment treatment on the cut voice. Because the HMM method is more robust and adaptable than the DTW approach, it will be used in this research to construct with the spoken English voice recognition system on the MATLAB environment. Furthermore, the system recognises isolated words in some English speech using a GUI graphical interface. The study findings show that the suggested artificial intelligence model in this article has a positive impact.

\section{Problem Statement}

The demand for high-quality English skills is increasing as our country's economy and society improve. The collection of educational materials and the invention of educational paradigms are both issues in the traditional English teaching system. Scholars and researchers are currently focusing their study on online teaching on promoting materials and reproducing conventional teaching patterns, which is not helpful for digital English education. This paper employs artificial intelligence algorithms to design a unique English network education system to remedy the aforementioned issue.

\section{Proposed Work}

In this section, the new English teaching methodology for university students is incorporated in a hierarchical method. Initially, the dataset is preprocessed using normalization, and the features of teaching methods are extracted using principal component analysis. The $k$-means clustering algorithm is used for classification of data, the student performance is evaluated using the whale optimization algorithm, and for the students who utilise the platform, their academic score performance is improved compared to the other students who do not utilise the platform. It also improves the autonomous learning abilities of the student (Figure 1).

4.1. Input Dataset. The learning patterns of 420 college students in Hanoi during the first 2 weeks of university shutdown due to COVID-19 were studied in this database. There are three main categories of items in the data: individual demographics, such as family socioeconomic status (SES), school type, and functional status; educators' learning practices, hour shifts of teaching before and during time frame of school detention, with or without the help of others; and educators' perceptions of their self-learning during in the closing schools. It also contains a questionnaire to assess the integration of online courses with sustainability themes throughout this period.

4.2. Data Preprocessing. The SA system analyzes the gathered data to be processed during this step. There are six stages in all.

(i) Tokenization: the tokenize function in $\mathrm{R}$ is used to break down student responses into tokens or words.

(ii) Lowercasing: to make matching terms in student responses to words in the NRC Emotion Lexicon easier, all characters are changed to lower case. The tm map function from R's tm packages is used to complete this phase.

(iii) Normalization: abbreviated information is normalized by mapping it to commonly used Internet slang terms using a dictionary. For example, "awsm" and "gud" are plotted to "awesome" and "good" correspondingly.

(iv) Stemming: words in student comments are transformed to their root word using the tm_map function in R's Snowball C package to make word matching easier. "Moving," "moved," and "movement," for example, are all transformed to "move."

(v) Removal of irrelevant content: to enhance system reaction speed and efficacy, unnecessary punctuation and stop words are eliminated. 


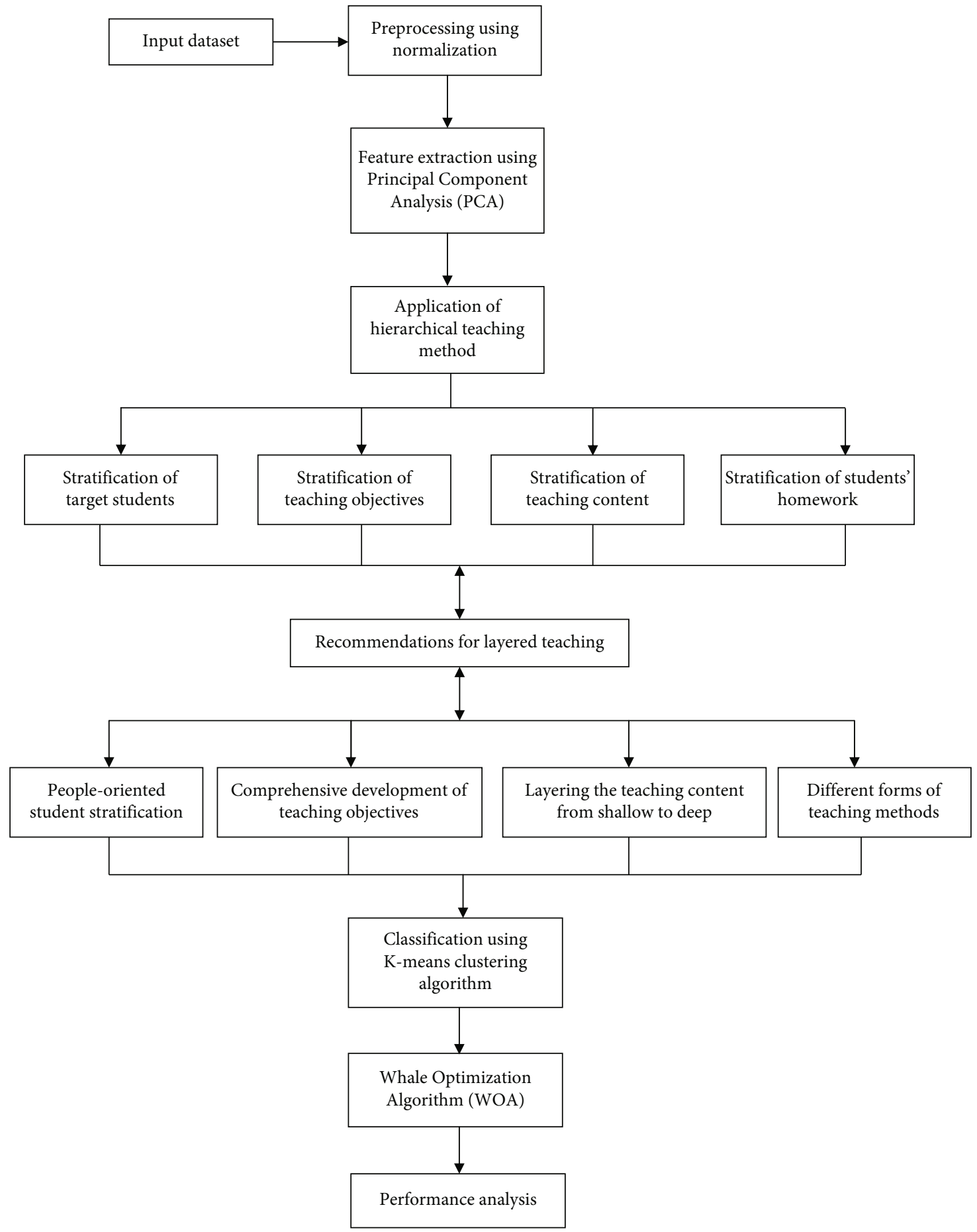

FIGURE 1: Flow of proposed method.

(vi) Transliteration: the content is transliterated to use the Google Transliterate API in order to solve the problem of mixed language in student responses.

4.3. Principal Component Analysis (PCA). As a statistical analysis technique, principal component analysis transforms original indications into many comprehensive indicators. Its primary purpose is to decrease the size of the object. Assume there are $n$ geographic samples, with $p$ variable descriptions for each sample. Following that, we obtain a geographic data matrix.

$$
X=\left[\begin{array}{cccc}
x_{11} & x_{12} & \cdots & x_{1 p} \\
x_{21} & x_{22} & \cdots & x_{2 p} \\
M & M & \cdots & M \\
x_{n 1} & x_{n 2} & \cdots & x_{n p}
\end{array}\right]
$$


How can I determine the internal regularity of a geographic object with a large amount of variable data? We solve this issue in $p$-dimensional space; however, this kind of practice is very difficult. As a result, we must decrease the dimension by replacing numerous indications with a few comprehensive ones. However, how can these comprehensive indications be obtained? The easiest approach is to utilise the original indicator's linear model.

Let $x_{1} x_{2}, \cdots x_{i}$ be the original variable indicators $x_{1} x_{2}$, $\cdots z_{m}(m \leq p)$ which are their comprehensive indicators (i.e., new variable indicators), then

$$
\left\{\begin{array}{l}
x_{1}-l_{11} x_{1}+l_{12} x_{2}+\cdots+l_{1 p} x_{p}, \\
x_{2}-l_{21} x_{1}+l_{22} x_{2}+\cdots+l_{2 p} x_{p}, \\
\cdots \cdots \cdots \\
z_{m}-l_{m 1} x_{1}+l_{m 2} x_{2}+\cdots+l_{m p} x_{p}
\end{array} .\right.
$$

$z_{1}$ is the linear combination of $x_{1} x_{2}, \cdots x_{p}$ satisfying the biggest variance; $z_{2}$ is the linear combination of $x_{1} x_{2}, \cdots x_{p}$ satisfying the biggest variance and unrelated with $z_{1}$; and $z_{m}$ is a linear combination of $x_{1} x_{2}, \cdots x_{p}$ satisfying the biggest variance and unrelated with $z_{1} z_{2}, \cdots z_{m-1}$

The new indicator $z_{1}(i=1,2, \cdots, m)$ is the principal component of the original indicator $x_{j}(j=1,2, \cdots, p)$. The largest percentage in the total variance is $z_{1}$, and then, variance of $z_{1} z_{2}, \cdots z_{m}$ are successive reductions. We often choose a few in the front of the maximum principal components. The aim is to reduce the index, but to also make clear the main contradiction, we simplify the relationship between indicators.

Based on the above analysis, to obtain the principal component, we determine the load $l_{i j}(i=1,2, \cdots m ; j=1,2, \cdots$, $p)$ of the original indicator $x_{j}(j=1,2, . ., p)$ in the principal component $z_{j}(i=1,2, \ldots, m)$. They are eigenvectors corresponding to $m$ larger eigenvalues of correlation matrix of $x_{1} x_{2}, \cdots x_{p}$.

4.4. Hierarchical Teaching Method. According to the characteristics and hobbies of the students, the hierarchical teaching method is designed.

According to the current English level, the teaching plan and goal should be developed and help students find their own English learning methods. The major problem for a teacher is how to improve the teaching level which is the first problem we need to solve and one of the topics worth studying. Therefore, this paper expounds the analysis and discussion on the application of the hierarchical teaching method in college English teaching, which is only for reference.

4.4.1. Stratification of Target Students. This teaching method is an education teaching method, which helps teachers play a leading role in teaching, to help improve students' enthusiasm for learning. Moreover, this kind of teaching method emphasizes the development of students' personality and self-satisfaction, and it can improve students' interest and motivation in learning English, so as to achieve the goal of helping students to learn and improve the teaching effect. Therefore, from the beginning of students entering the campus, the school must divide students into multiple levels according to their personality differences and actual English level and conduct targeted teaching. For example, we can first divide some students with good grades, solid basic knowledge, strong independent learning ability, and strong receptivity into group A. And for some students who have high interest in learning, lack of solid basic knowledge, and generally accept the ability, as long as the teacher carries out timely supervision and supervision, students who will make rapid progress will be divided into group B. Students with low self-learning ability, poor basic knowledge, weak receptivity, and no interest in learning and students who need teachers' constant supervision in learning were divided into group C. In this case, we can divide the students at these 3 levels into one group, so that the students in group A can supervise and urge the students in the other groups and help them in some difficult problems. Also, it can then be adjusted in a timely manner according to the development of the group. In this way, we can not only improve students' initiative in learning but also stimulate students' internal potential of some high overall teaching level.

4.4.2. Stratification of Teaching Objective. Through some investigations, it is found that students with good academic performance and enterprising spirit have set goals for learning, while some students with poor academic performance and no interest in learning have no corresponding goals. Therefore, we should set specific teaching and learning goals according to students at different levels and help students provide direction for learning, so that students are enthusiastic about learning.

4.4.3. Stratification of Teaching Content. The stratification of teaching content refers to the stratification of the difficulty of teaching course content. Every day, we routinely use these new words and new phrases in each course. Every level student must grasp this new content, and then for some more focus on knowledge and language points, just like every day's course in some relatively new and important knowledge, it is necessary to require students in group A and group B to master and digest it in a timely manner, so some of the students in group $\mathrm{C}$ ask them to make a general understanding of as much as they can. Finally, in terms of the teaching of some key and difficult knowledge, we do not require the students of group C, but rather require the students of group B, to understand and accept in general and require the students of group A to break through the difficult points and master them, which can help lay a solid foundation for future study.

4.4.4. Stratification of Students' Homework. English teaching is the same as that of other courses. Students cannot rely solely on the content in class, and it is difficult for them to master the knowledge points taught. Students of different levels are often given the same homework. Therefore, the application of stratified teaching should be stratified for students' homework. The amount and difficulty of homework given should also be treated differently and chosen 
appropriately so that each student's learning can be consolidated and developed in the best way. Stratified exercise and stratified examination of homework after class are helpful for teachers to correctly grasp students' learning situation, carry out individual teaching activities, and improve teaching quality in a large area.

4.4.5. The Recommendations for Layered Teaching Stratification. The theory of multiple intelligence reveals the principle that everyone can be talented, but the methods needed are different from those of talents. This requires our college English teachers to have confidence in students. To achieve a layered teaching of students in the true sense, the first is that teachers organize students to conduct a unified test, determine the actual level of each student, and prepare for follow-up teaching. Secondly, the students' learning ability and learning potential are discovered. This is mainly based on the students' learning habits and attitudes. At the same time, some students, although they have poor academic performance in the short term, have a serious attitude and will make rapid progress. In the end, they should make reasonable adjustments to the level of teaching based on the dynamic learning situation of students. The rationality of stratification is guaranteed.

\subsubsection{Comprehensive Development of Teaching Objectives.} The teaching goal is the guidance of the whole teaching process and also the ultimate goal and destination of the teaching. The stratification of teaching objectives should be based on the principle of "facing the whole and taking care of both ends," starting from strengthening basic development, cultivating individual abilities, and comprehensively improving overall quality and formulating goals that meet the needs of students at all levels. First of all, for students with good grades in English, they should focus on cultivating their knowledge points, develop their ability to comprehensively apply knowledge and expand their knowledge and vision. Secondly, students with intermediate English should listen. Speaking of reading and writing, to achieve the mastery of knowledge points, finally, students with poor English scores should focus on understanding English knowledge, especially word recognition and sentence understanding. The stratification of teaching objectives avoids the one-size-fitsall situation of traditional teaching, enhances the learning confidence of middle and lower level students, and realizes their self-positioning.

4.4.7. Layering the Teaching Content from Shallow to Deep. Traditional English teaching pays much attention to students' test scores, especially the test-oriented education. The teaching content is basically started from the test sites and rarely involves students' study skills, skills, ideas, and even personal behavior. College students are in a critical period of life development, and they have more spare time. If they are not interested in English learning, it is difficult to learn English by themselves, which causes a decline in the English performance. The stratification of teaching content under the new curriculum reform requires a certain degree of gradient and at the same time guides students' development potential. For example, in the teaching of English essay reading, you can first set a certain teaching situation, and you can also use the multimedia video and pictures to introduce the text and use the questioning method to read the short text; secondly, for the students with poor grades, just ask questions yes or no, but the students in the middle and upper reaches need to raise more difficult problems. The best questions can reflect a certain level of listening and language skills. Finally, students can cooperate with each other, that is, students with better grades. Middle school students ask questions, middle school students ask questions to downstream students, etc., while cooperating with each other, deepening the impression of each student and improving the teaching efficiency.

4.4.8. Different Forms of Teaching Methods. The so-called teaching method refers to the special teaching activities that teachers take to complete certain teaching goals. At this stage, teachers mostly use analytical methods, situational simulation methods, etc. Some teachers also use the college entrance examination as the sole guide and adopt the teaching methods such as "full house irrigation" and "one sentence hall," which lead to the passive learning process of students. The stratification of various forms of teaching methods first requires teachers to improve their comprehensive quality, including professional knowledge and personal accomplishment, and establish the concept of lifelong learning to better master modern teaching methods and shorten the gap with students. Secondly, teachers should be good at using modern teaching methods such as multimedia. After all, college students have their own electronic devices such as mobile phones and computers, and they can make good use of them to improve their English scores. For example, students with better English scores can encourage them to use their amateur English videos to try to translate themselves; students with poor English scores can be encouraged to listen to more English words and sentences, which could enhance your confidence.

4.5. Classification of K-Clustering Algorithm-Based WOA Method. It is essential to first build data sampling models of the constraining variables of the hierarchical teaching method in order to obtain an accurate evaluation of English teaching ability. To conduct a statistical study of the hierarchical teaching method, we combine nonlinear data $K$-means clustering-based WOA techniques with time series analysis methods. A collection of nonlinear time periods makes up the hierarchical teaching technique constraint index variable. A high-dimensional feature distribution space is created to describe the variable index distribution system of the standardized English assessment, with teacher level, learning facility investment, and policy relevancy level as the major provided recommendations of the restrained hierarchical teaching method.

For the analyzing of hierarchical teaching methods based on language education, a constricted parametric index analysis model is formed, a quantitative recursive analysis is used to compare hierarchical teaching methods depending on big data knowledge analysis model in terms of improving the 


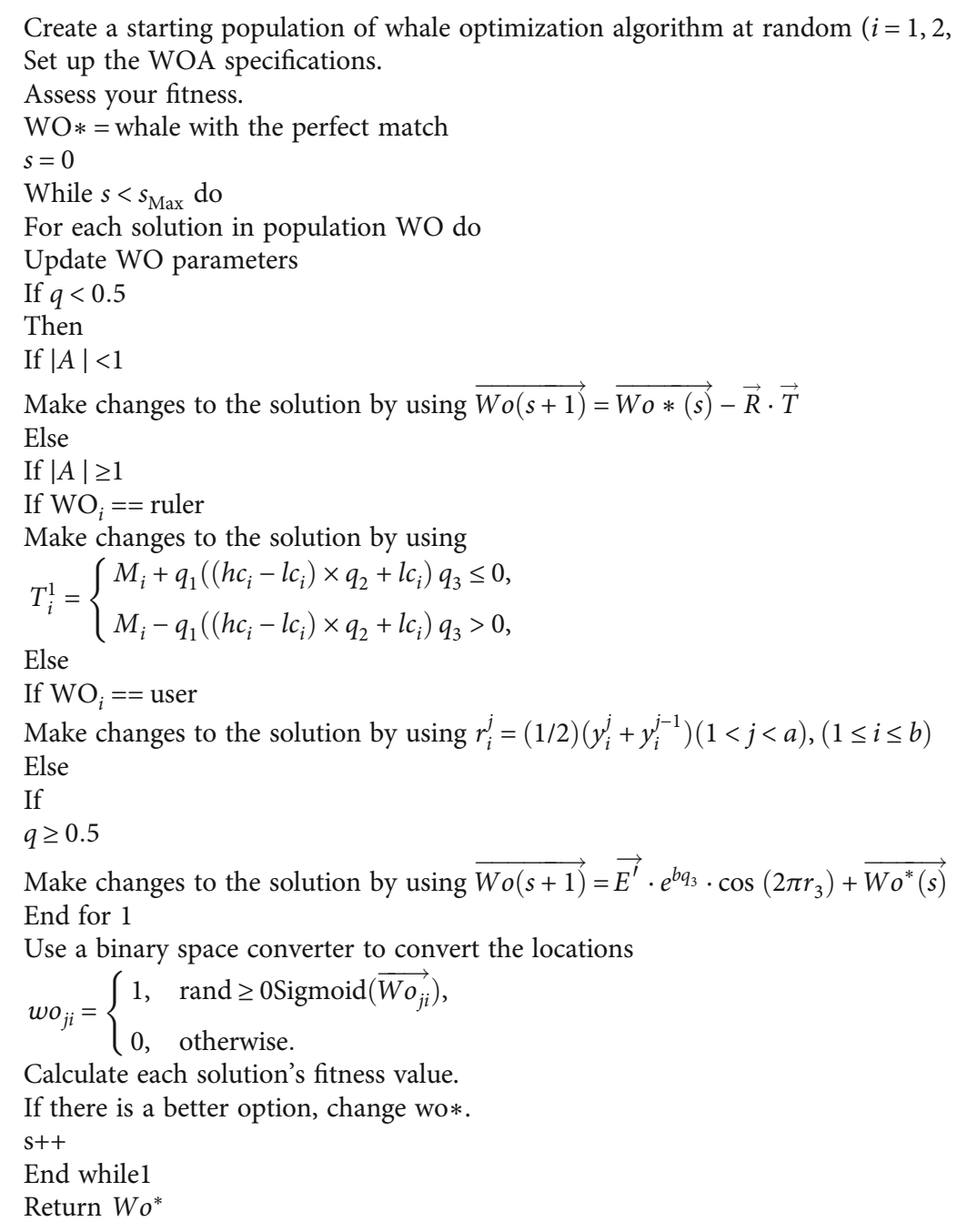

Pseudocode 1: Pseudocode $K$-cluster with WOA algorithm.

capacity of quantitative evaluation of English training level, and $K$-means clustering and WOA are suggested based on large datasets, and the issue of assessing hierarchical teaching methods is transformed into addressing the $K$-means clustering optimal solution as a least square assessment problem using the hierarchical teaching method evaluation technique based on the information. The hierarchical teaching method values are calculated as

$$
l_{\mathrm{los}}=\min \left\{\left\|z_{j}-v_{j}\right\|\right\} .
$$

The value of the cluster center of each cluster after each cycle of iterations is recalibrated using the following formula.

$$
l_{\operatorname{los}}(k+1)=\frac{1}{M_{i}} \sum z_{j} .
$$

we establish a hierarchical tree, then apply a big data analysis technique to determine the PCA feature quantity of the hierarchical teaching method, and then use the $k$-means closeness fill method for solving the similarity of teaching allocation and combining the linear regression feature $k$ means method with index parametric cluster formation and integration; the outcome of teaching resource $k$-means expression is acquired, and the corresponding teaching allocation of the resource plan is compiled via index parameter clustering and WOA, resulting in hierarchical teaching method evaluation optimization.

4.6. Whale Optimization Algorithm (WOA). For continuous optimization problems, WOA is a swarm intelligence method. It has been demonstrated that this method outperforms or is equivalent to certain current algorithmic strategies. The hunting activity of humpback whales has served as an inspiration for WOA. Each solution in WOA is regarded as a whale. In this approach, a whale attempts to fill a new spot in the search area using the best element of the group as a benchmark. The whales have two systems for searching for prey and attacking. The prey are ringed in the first, and bubble nets are created in the second. In terms of optimization, as whales hunt for prey, they explore the 


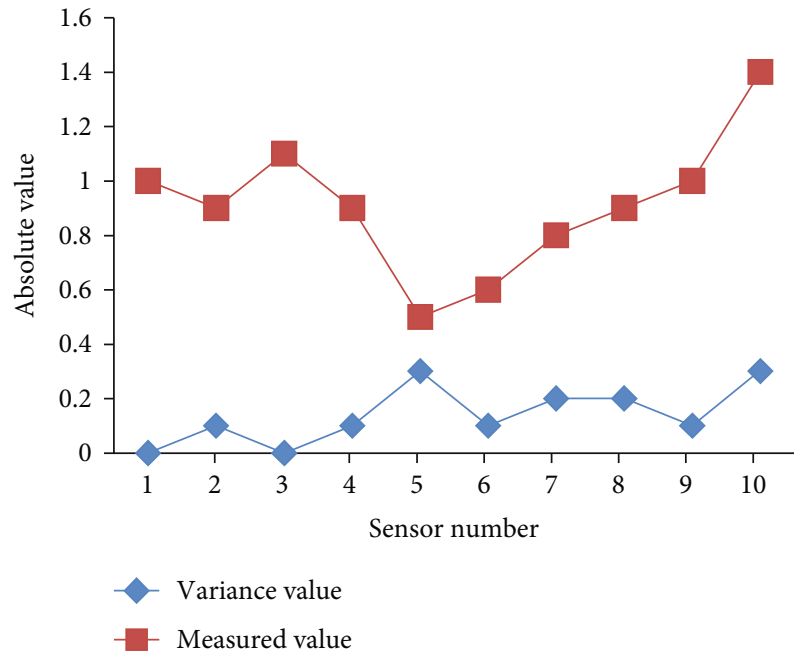

FIGURE 2: Measured value vs. variance value.

search area, and exploitation happens during the attack behavior.

A set of random solutions is used to start the WOA algorithm. At the end of each iteration, search agents compare their positions to a randomly picked search agent or the best solution found so far. In order to facilitate exploration and exploitation, the $s$ parameter is reduced from 2 to 0 . When $|A|>1$, a random search agent is picked, while when $|A|<1$, the best solution is chosen for updating the search agents' positions. WOA can flip between a spiral and a circular movement depending on the value of $q$. Finally, the WOA algorithm is ended when a termination requirement is satisfied.

\section{Performance Analysis}

A simulated experiment is performed on the technique to evaluate the efficacy of the revised method for the experimental findings and analysis. MATLAB was chosen as the tool.

The optimum result that is evaluated using MATLAB simulation tool is 1.0049 when using to conduct $k$-means processing on the chosen big dataset. Figure 2 shows the measurement findings as well as the sensor variance parameters.

The comparative accuracy analysis result is shown in Figure 3. In comparison to other algorithms, our suggested approach is the most effective in terms of accuracy.

Figure 4 shows the graph for error rate (\%) for the existing and the proposed method. It demonstrates that our suggested method has a lower error rate when compared to existing strategies.

Figure 5 shows the comparative analysis of performance ratio (\%) for the existing and the proposed method. It demonstrates that our suggested method has a higher performance ratio when compared to other existing methods.

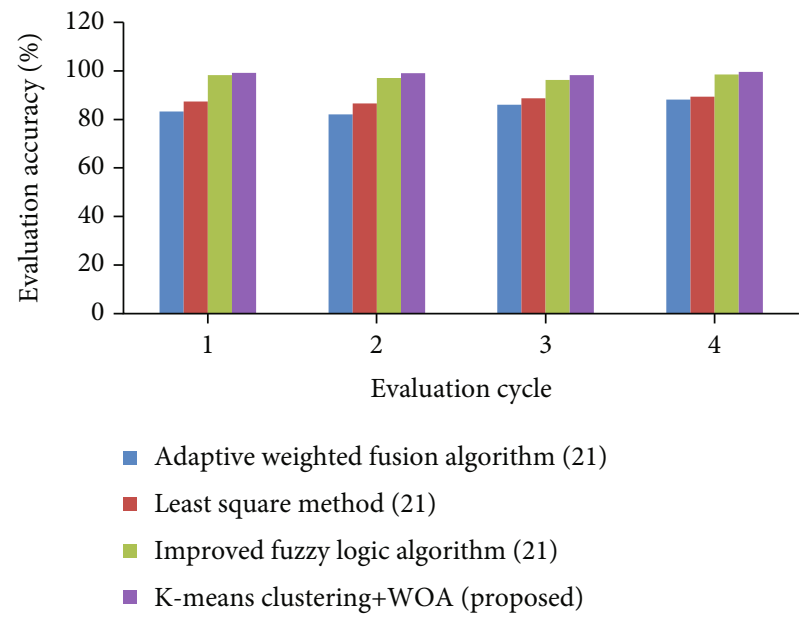

Figure 3: Comparison of accuracy.

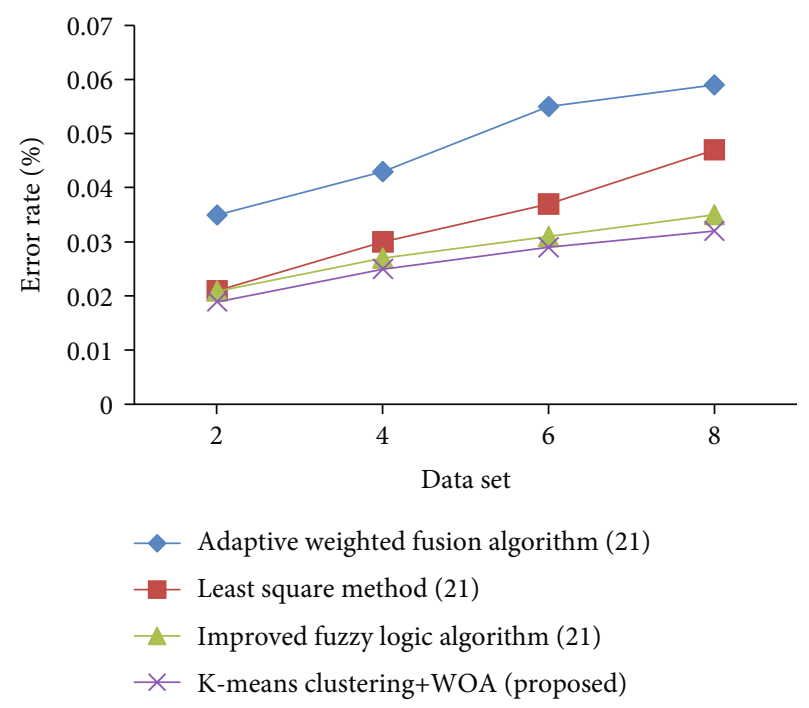

FIGURE 4: Error rate vs. dataset.

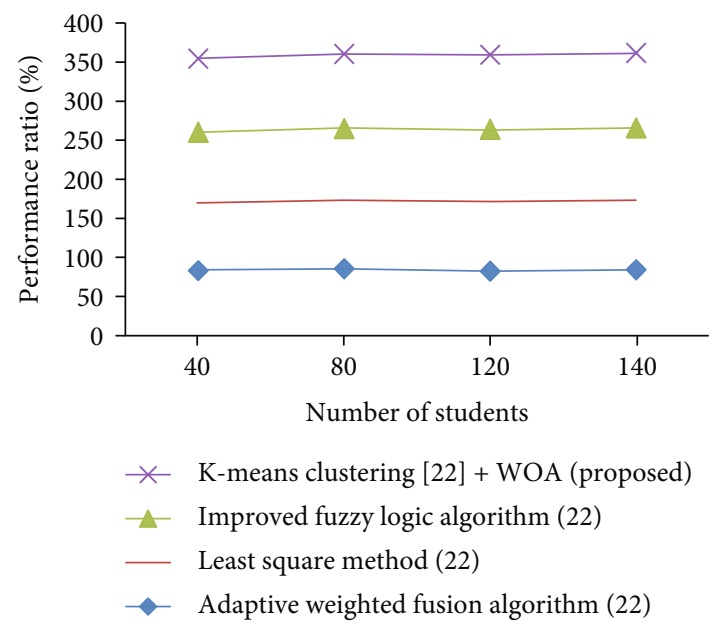

FIGURE 5: Performance ratio vs. number of students. 


\section{Conclusions}

This article evaluates the optimization model for assessing hierarchical teaching methods, proposes a hierarchical teaching method based on big data $K$-means clustering pulse WOS, develops a constraint parameter index analytical framework for assessing and analyzing hierarchical teaching methods, and employs a quantitative iterative analysis approach. The PCA feature extraction of the hierarchical teaching method restriction feature information is realized by the big data knowledge model analysis of hierarchical teaching method evaluation, which manages to combine the big data knowledge with the $K$-means clustering method to improve the clustering and classification WOS method to improve the integration of the index parameters of the hierarchical teaching method. According to research, the approach presented in this article is more accurate in assessing hierarchical teaching methods and increases the efficiency with which English teaching resources are used.

\section{Data Availability}

The data used to support the findings of this paper are available upon request to the corresponding author.

\section{Conflicts of Interest}

The authors declare that there are no conflicts of interest regarding the publication of this paper.

\section{References}

[1] M. Sun and Y. Li, "Eco-environment construction of English teaching using artificial intelligence under big data environment," IEEE Access, vol. 8, pp. 193955-193965, 2020.

[2] Y. Bin and D. Mandal, "English teaching practice based on artificial intelligence technology," Journal of Intelligent \& Fuzzy Systems, vol. 37, no. 3, pp. 3381-3391, 2019.

[3] L. Zhao, L. Chen, Q. Liu, M. Zhang, and H. Copland, “Artificial intelligence-based platform for online teaching management systems," Journal of Intelligent \& Fuzzy Systems, vol. 37, no. 1, pp. 45-51, 2019.

[4] N. S. Kumar, "Implementation of artificial intelligence in imparting education and evaluating student performance," Journal of Artificial Intelligence, vol. 1, no. 1, pp. 1-9, 2019.

[5] Z. Sun, M. Anbarasan, and D. Praveen Kumar, "Design of online intelligent English teaching platform based on artificial intelligence techniques," Computational Intelligence, vol. 37, no. 3, pp. 1166-1180, 2021.

[6] L. Zhao and H. Yu, "Retracted article: a wireless network remote monitoring method driven by artificial intelligence," International Journal of Computers and Applications, vol. 40, pp. 1-9, 2020.

[7] P. Li, Y. Ning, and H. Fang, "Artificial intelligence translation under the influence of multimedia teaching to study English learning mode," The International Journal of Electrical Engineering \& Education, vol. 13, no. article 002072092098352, 2021.

[8] D. Juan and Y. Hong Wei, "Particle swarm optimization neural network for research on artificial intelligence college English classroom teaching framework," Journal of Intelligent \& Fuzzy Systems, vol. 40, no. 2, pp. 3655-3667, 2021.
[9] Y. Chen, "College English teaching quality evaluation system based on information fusion and optimized RBF neural network decision algorithm," Journal of Sensors, vol. 2021, 9 pages, 2021.

[10] Y. Lou, "Storage and allocation of English teaching resources based on k-nearest neighbor algorithm," International Journal of Emerging Technologies in Learning (iJET), vol. 14, no. 17, pp. 102-113, 2019.

[11] T. Zhang, "Application of AI-based real-time gesture recognition and embedded system in the design of English major teaching," Wireless Networks, vol. 3, pp. 1-13, 2021.

[12] J. Guo, "Empirical analysis for English teaching integration and optimization based on big data mining technology," in Proceedings of the 2020 International Conference on Computers, Information Processing and Advanced Education, pp. 504-508, Information Processing and Advanced Education. CIPEA, 2020.

[13] H. Li, "Text recognition and classification of English teaching content based on SVM," Journal of Intelligent \& Fuzzy Systems, pp. 1-11, 2020.

[14] X. Jiang, "Online English teaching course score analysis based on decision tree mining algorithm," Complexity, vol. 2021, 10 pages, 2021.

[15] X. Shuoyan, "Research on college English teaching model based on computer network technology," Journal of Intelligent \& Fuzzy Systems, vol. 2, pp. 1-5, 2017.

[16] Y. Quan, "Development of computer aided classroom teaching system based on machine learning prediction and artificial intelligence KNN algorithm," Journal of Intelligent \& Fuzzy Systems, vol. 39, no. 2, pp. 1879-1890, 2020.

[17] W. Dan, "Intelligent English resource recommendation and teaching effect based on symmetric SDAE collaborative filtering algorithm," Journal of Ambient Intelligence and Humanized Computing, vol. 3, pp. 1-11, 2021.

[18] M. Wanni, "Research on English grammar recognition system based on combination of genetic algorithm and KNN algorithm," Journal of Intelligent \& Fuzzy Systems, vol. 38, no. 6, pp. 7291-7302, 2020.

[19] X. Zhao, "Mobile english teaching system based on adaptive algorithm," International journal of emerging technologies in learning (ijet), vol. 13, no. 8, pp. 64-77, 2018.

[20] D. Ran, W. Yingli, and Q. Haoxin, "Artificial intelligence speech recognition model for correcting spoken English teaching," Journal of Intelligent \& Fuzzy Systems, vol. 40, no. 2, pp. 3513-3524, 2021. 